\title{
SECONDs Administration Guidelines: A Fast Tool to Assess Consciousness in Brain-injured Patients
}

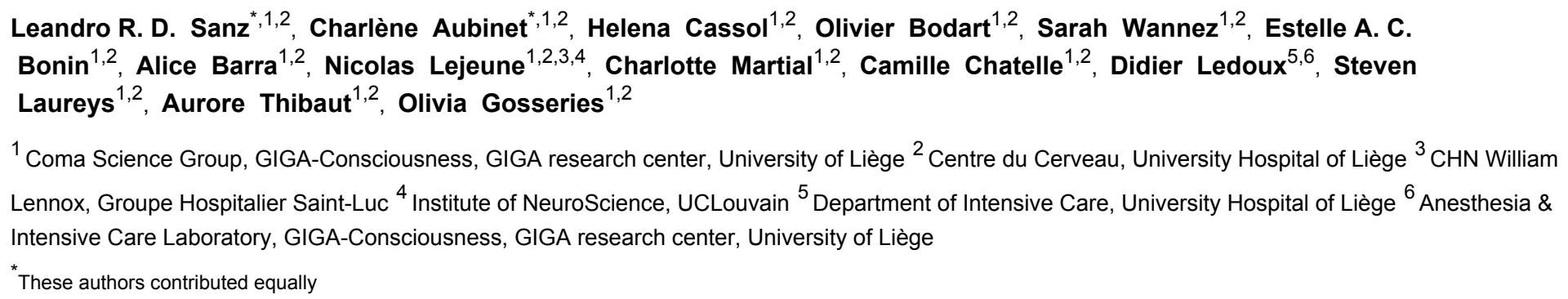

\section{Corresponding Author}

Leandro R. D. Sanz

Leandro.Sanz@uliege.be

\section{Citation}

Sanz, L.R.D., Aubinet, C., Cassol, H., Bodart, O., Wannez, S., Bonin, E.A.C., Barra, A., Lejeune, N., Martial, C., Chatelle, C., Ledoux, D., Laureys, S., Thibaut, A., Gosseries, O. SECONDs Administration Guidelines: A Fast Tool to Assess Consciousness in Brain-injured Patients. J. Vis. Exp. (168), e61968, doi:10.3791/61968 (2021).

\section{Date Published}

February 6, 2021

DOI

$10.3791 / 61968$

URL

jove.com/video/61968

\section{Abstract}

Establishing an accurate diagnosis is crucial for patients with disorders of consciousness (DoC) following a severe brain injury. The Coma Recovery ScaleRevised (CRS-R) is the recommended behavioral scale for assessing the level of consciousness among these patients, but its long duration of administration is a major hurdle in clinical settings. The Simplified Evaluation of CONsciousness Disorders (SECONDs) is a shorter scale that was developed to tackle this issue. It consists of six mandatory items, observation, command-following, visual pursuit, visual fixation, oriented behaviors, and arousal, and two conditional items, communication and localization to pain. The score ranges between 0 and 8 and corresponds to a specific diagnosis (i.e., coma, unresponsive wakefulness syndrome, minimally conscious state minus/plus, or emergence from the minimally conscious state). A first validation study on patients with prolonged DoC showed high concurrent validity and intraand inter-rater reliability. The SECONDs requires less training than the CRS-R and its administration lasts about 7 minutes (interquartile range: 5-9 minutes). An additional index score allows the more precise tracking of a patient's behavioral fluctuation or evolution over time. The SECONDs is therefore a fast and valid tool for assessing the level of consciousness in patients with severe brain injury. It can easily be used by healthcare staff and implemented in time-constrained clinical settings, such as intensive care units, to help decrease misdiagnosis rates and to optimize treatment decisions. These administration guidelines provide detailed instructions for administering the SECONDs in a standardized and reproducible manner, which is an essential requirement for achieving a reliable diagnosis. 


\section{Introduction}

Disorders of consciousness (DoC) are characterized by a prolonged impaired awareness following a severe brain injury ${ }^{1}$. When patients recover eye-opening after a period of coma but do not display any reproducible signs of awareness of their self or their surroundings, they are diagnosed with unresponsive wakefulness syndrome (UWS; formerly known as vegetative state $)^{2,3}$. As patients recover unambiguous behavioral signs of awareness evidence toward their self or their environment, they are considered to have transitioned to the minimally conscious state (MCS) ${ }^{4}$. Because of its clinical heterogeneity, the MCS has been further sub-categorized into MCS minus (MCS-) and MCS plus (MCS +$)^{5}$. MCSpatients only demonstrate low-level signs of consciousness, such as visual fixation and pursuit, automatic motor reactions, and localization to noxious stimulation, while MCS+ patients demonstrate higher-order language-related behaviors, such as following simple commands, intelligibly verbalizing, and/ or intentionally communicating ${ }^{5}$. These patients emerge from the MCS (EMCS) once they regain the ability to functionally communicate (i.e., reliably use a "yes/no" code) and/or to use objects such as a comb or a cup ${ }^{4}$. A differential diagnosis has to be made between these clinical entities and the locked-in syndrome (LIS), a state of severe paralysis with preserved cognitive functions that can be confused with a state of impaired consciousness ${ }^{6}$. Previous studies have further shown the importance of correctly diagnosing the DoC to improve patient daily management (e.g., pain treatment or neurostimulation protocols ${ }^{7,8}$ ), determine longterm prognosis ${ }^{9}$, and support end-of-life decisions ${ }^{10}$.

Nevertheless, establishing an accurate diagnosis is challenging $11,12,13,14$ and there is a substantial rate of misdiagnosis when relying only on medical consensus rather than validated tools ${ }^{15}$. Several behavioral diagnostic scales have been elaborated in the last decades. The Coma Recovery Scale-Revised (CRS-R) ${ }^{16}$ is currently considered the most efficient scale, as it includes all MCS criteria and has an excellent content validity ${ }^{17}$. It has, however, several drawbacks, including a total score that does not correspond to a single behavioral diagnosis, a protocol featuring repeated painful stimulations (possibly reducing patient motivation), a learning curve requiring extensive examiner training, and a time-consuming administration procedure ${ }^{18,19,20}$. These various aspects constitute an even bigger issue given the need for repeated assessments (i.e., at least five) in a short time period (e.g., 10 days), as recently highlighted to avoid misdiagnoses within this population ${ }^{21}$. The time that should be allocated by clinicians to perform the CRS-R is rarely available in the clinical reality and long assessments can increase patient fatigability and lack of compliance ${ }^{22}$.

These guidelines describe the administration of a recently validated scale, the Simplified Evaluation of CONsciousness Disorders (SECONDs), to assess the level of consciousness in patients with severe brain injury ${ }^{22}$. This scale includes eight items: observation, command-following, communication (intentional or functional - conditional item), visual pursuit, visual fixation, localization to pain (conditional item), oriented behaviors, and arousal. Compared to the CRS-R, less training is required for the examiners and the resulting score is directly related to a level of consciousness, ranging from EMCS (8), MCS+ (6-7), MCS- (2-5), UWS (1), to coma (0). The items were selected either because they were the most frequently observed in the CRS-R among MCS patients ${ }^{23}$ or due to their importance for the diagnosis of EMCS (i.e., functional communication $)^{16}$. These administration 
guidelines aim to provide visual standardized instructions and hands-on targeted advices to properly administer and score each item of the scale.

\section{Protocol}

The following protocol and its validation study have been approved by the Ethics Committee of the University and University Hospital of Liège (reference 2017-297) and comply with the institution's guidelines on human research. All subjects (or their legal surrogates) who contributed to the elaboration and validation of the scale gave their written informed consent to participate. All personal data were processed according to the General Data Protection Regulation.

This scale has been developed with the aim to provide a comprehensive diagnostic assessment tool that would allow the examination of a large spectrum of patients with varying levels of consciousness. Like the CRS-R on which it is based, the SECONDs does not require specific inclusion or exclusion criteria to be administered. However, it has been validated in a population of patients with prolonged DoC, with predefined inclusion and exclusion criteria (see Representative Results).

\section{The Simplified Evaluation of CONsciousness Disorders (SECONDs)}

1. Before starting the behavioral examination, adjust the lighting of the room to be adequate to perform the exam and ensure the patient is comfortably positioned with the four limbs exposed and the head oriented as straight as possible.

2. Turn off any $\mathrm{TV}$, radio, or other potentially distracting stimuli.
3. Note any recent changes in medication in the patient's current treatment regimen, with particular attention to sedative and psychoactive drugs.

4. Select a mirror of a minimum recommended $10 \mathrm{~cm} \times 10$ $\mathrm{cm}$ size for a square-shaped mirror or a $10 \mathrm{~cm}$ in diameter for a round-shaped one.

NOTE: Items should be administered sequentially from A to $\mathrm{H}$ : observation, command-following (score 6), communication (conditional, scores 7 and 8), visual pursuit (score 4), visual fixation (score 3), localization to pain (conditional, score 2), oriented behaviors (score 5 ) and arousal (scores 1 and 0 ). This sequence does not follow the scores of the items ordinally. This order was designed to optimize the allocation of the patient's attentional capacities and reduce administration duration. Communication and localization to pain are conditional items and must be administered only under certain conditions (see corresponding sections). The final score corresponds to the score of the highest succeeded item and directly reflects the diagnosis. An additional index score can be calculated to obtain a more accurate measure of the observed behaviors and allows following the evolution of the patient over time (see corresponding section).

\section{Observation (A)}

1. Stimulation: at any time during the assessment, if no sustained eye-opening is observed or if the patients stops following commands for at least one minute, administer auditory (i.e., use patient's own name, clapping hands), tactile (i.e., CRS-R arousal facilitation protocol $^{16}$ ), or noxious stimulation (i.e., pressure on fingernail bed) to arouse the patient. 
2. Observe the patient for one minute and report spontaneous behaviors.

NOTE: Pay attention to vocalizations, spontaneous movements of the four limbs, head, lips, or eyes, as well as spontaneous interactions with the environment.

3. During the entire assessment, promote eye-opening before testing each item if needed. Observe the patient and report the presence of eye-opening, either spontaneously or in response to stimulation, as well as the presence of self-oriented behaviors or behaviors oriented toward the environment. See sections 9 and 10 for detailed scoring guidelines of oriented behaviors and arousal.

NOTE: If eye-opening is not possible, oculomotor command-following, visual pursuit, and visual fixation (see sections $4,6,7$ ) should be assessed by opening the patient's eyes manually. See section 10 (arousal) for detailed scoring criteria for eye-opening.

\section{Command-following (B)}

1. Select three simple movements that were not observed as spontaneously repetitive during the observation period. Appropriate examples include: 'Move your hand', 'Turn your head', 'Blink twice', 'Look at [object or person]', 'Look up/down', 'Open/close your mouth', 'Stick out your tongue', 'Say a word/make a sound'. Adapt the selection of commands to the physical abilities of the patient. In cases of suspected LIS, relate at least one command to eye movements.

2. Test each command in three trials, with a 10-second interval between trials. A command may be repeated once within the same trial to increase patient motivation. If the two first commands are successfully performed ( $3 / 3$ trials for both commands with accurate responses), the third command does not have to be administered.

3. In cases of known or suspected deafness, administer written commands. If the patient does not react to any of the oral commands, test at least one written command.

4. Score "6" for command-following if the patient accurately responds to $2 / 3$ trials for at least one command. To be scored, the response has to be clear and must appear within 10 seconds following the command prompt (and not spontaneously). All unclear, ambiguous responses or reflexive movements due to spasms or grasping must not be scored. Report the commands used on the scoring sheet, as well as the number of successful trials.

\section{Communication (conditional) (C)}

NOTE: Perform a communication test if at least two distinct responses to command are successfully performed (i.e., at least $2 / 3$ for two commands) or if the patient can express a "yes" and a "no", either verbally, through gestures, eye movements or writing, spontaneously or not.

1. If no verbal response can be produced, base the communication code on previously identified motor responses. Clearly explain the code to the patient before starting (e.g., thumbs-up for a "yes" and thumbs-down for a "no") and employ the most frequently used code for this patient, if any. The examiner can remind the code to be used to the patient before each question.

NOTE: It is necessary to use two distinct responses; the absence of movement cannot be used for a "yes" or a "no".

2. First ask 5 binary autobiographical questions - (1) 'Is your name [incorrect name]?' (2) 'Are you born in [correct birth 
year]?' (3) 'Is your name [correct name]?' (4) 'Are you born in [incorrect birth year]?' (5) 'Do you have children?'

3. If the patient fails to correctly answer the autobiographical questions, ask the following binary situational questions: (1) 'Are we in [place: hospital, home or else]?' (2) 'Am I wearing a hat?' (3) 'Are we at the swimming pool?' (4) 'Am I touching your hand?' (Touch) (5) 'Am I touching your face?' (Do not touch).

4. Score "7" for intentional communication if the patient responds to at least three out of five questions from one question set, regardless of accuracy. Score "8" for functional communication if the patient correctly responds to the five questions from one question set (either autobiographical or situational). Report the nature of the "yes/no" code, the modality (verbal, written) and the type (autobiographical, situational) of the questions used, the number of responses and the number of correct ones.

NOTE: Question sets must be considered separately, and correct answers from distinct question sets cannot be added up when scoring the item.

\section{Visual pursuit (D)}

1. To assign a visual pursuit score, move silently around the bed while observing whether the patient's gaze spontaneously and clearly follows this movement during at least two seconds in two different directions.

2. If a clear pursuit is not spontaneously observed, position the mirror about $30 \mathrm{~cm}$ in front of the patient's face.

3. After confirming that the patient can see their reflection, move the mirror slowly from left to right (or right to left, depending on the initial position of the patient's eyes), right to the left, top to bottom, and bottom to top for at least four seconds per movement.

4. Score a "4" for visual pursuit if an uninterrupted visual pursuit is observed in two different directions for at least two seconds. Report the number of observed pursuits on each axis, the type of stimulus used (spontaneous, mirror), and whether manual eye-opening was employed.

\section{Visual fixation (E)}

1. To score visual fixation, enter the patient's field of view and observe whether the patient's gaze spontaneously fixates on the examiner for at least two seconds in two different visual quadrants by turning toward the examiner (performing a saccadic eye movement).

2. If no clear and spontaneous visual fixations are observed, present the mirror about $30 \mathrm{~cm}$ away from the patient's face in all four quadrants of the patient's visual field, but not in the axis of their gaze, for at least four seconds per quadrant.

3. Score a "3" for visual fixation if two fixations are observed, either spontaneously or induced by the mirror. A clear change of gaze orientation toward the mirror (or examiner) followed by a fixation of at least two seconds should be observed. Report the quadrants in which the patient showed the fixations, as well as the type of the stimulus used (spontaneous, mirror), and whether manual eye-opening was employed.

\section{Localization to pain (conditional) (F)}

NOTE: Test localization to pain only if the patient did not demonstrate command-following (score of 5 or below) 
1. Place a pen or pencil on the patient's fingernail bed for five seconds before instructing the patient to 'Remove your hand to avoid the pain'.

2. If the patient does not remove the hand within the next five seconds, administer pressure on the nail bed with the pen or pencil for five seconds. One trial should be performed on each hand. If the patient removed the hand after the warning, do not apply pressure and directly proceed to the other hand and repeat the warning.

3. Score "2" for localization to pain if, during at least one of the two trials, the non-stimulated hand of the patient clearly touches the stimulated hand. If the patient clearly removes the pain-stimulated hand after the warning but before the stimulation on both trials (anticipation response), score "6" for commandfollowing. Report the side ( $L$ or $R$ ) of each observed localization and anticipation response (always report stimulated side).

\section{Oriented behaviors (G)}

1. To assess oriented behaviors, perform continuous observation as described in 2.3. and score oriented behaviors by considering all of the behaviors observed during the entire examination. These behaviors may include (but are not limited to) scratching themselves, grabbing the bed sheets, holding the bed, pulling on the clothes/tracheostomy/gastrostomy/ catheter, placing the hand on the mouth to cough, smiling/laughing/crying contextually, stereotyped verbal response or gesture when spoken to (e.g., grunt, head nod or thumbs up), or any other automatic non-reflexive behaviors.

NOTE: Yawning may not be scored as an automatic nonreflexive behavior.
2. Score "5" for oriented behaviors if the patient presents at least one clearly observed oriented behavior. Report the type and the number of times each behavior is observed.

\section{Arousal (H)}

1. To assess arousal, perform continuous bbservation as described in 2.3. and score "0" for no arousal if, during the entire evaluation, the patient never opened the eyes, with or without stimulation (including nociceptive stimulation). Score "1" for arousal if the patient opened the eyes at least once during the assessment, either spontaneously or following stimulation. Report the approximate percentage of time the eyes were open throughout the examination $(0-25 \% ; 25-50 \% ; 50-75 \%$; $75-100 \%)$.

2. Specify if the eye-opening happened spontaneously or following a noxious, tactile, or auditory stimulation, as well as the number of stimulations of each type that were administered

\section{Additional index}

NOTE: An additional index score has been developed to give a more accurate account of the behaviors observed during the assessment, allowing monitoring of a patient's level of consciousness over time. For each successful item, specific index points correspond to the type of behavior observed (see Table 1).

1. Calculate the additional index score by adding up the points earned in each item tested during the assessment. This measure ranging from 0 to 100 is independent from the final score and diagnosis but is designed to more precisely follow the evolution of a patient who may 
exhibit subtle clinical changes without modification of the

diagnosis across repeated assessments.

\begin{tabular}{|c|c|c|c|c|}
\hline Diagnosis & Score & Item & Sub-item & Additional index points \\
\hline EMCS & 8 & \multirow[t]{4}{*}{ Communication* } & 5 answers (accurate) & 29 \\
\hline \multirow[t]{7}{*}{ MCS+ } & \multirow[t]{3}{*}{7} & & $\begin{array}{c}3 \text { or } 4 \text { answers } \\
\text { (accurate) }\end{array}$ & 21 \\
\hline & & & $\begin{array}{c}5 \text { answers } \\
\text { (inaccurate OK) }\end{array}$ & 14 \\
\hline & & & $\begin{array}{l}3 \text { or } 4 \text { answers } \\
\text { (inaccurate OK) }\end{array}$ & 7 \\
\hline & \multirow[t]{4}{*}{6} & \multirow[t]{4}{*}{ Command-following } & 2 commands $3 / 3$ & 24 \\
\hline & & & 2 commands $2 / 3$ & 18 \\
\hline & & & 1 command $3 / 3$ & 12 \\
\hline & & & 1 command $2 / 3$ & 6 \\
\hline \multirow[t]{11}{*}{ MCS- } & \multirow[t]{3}{*}{5} & \multirow[t]{3}{*}{ Oriented behaviors } & $\begin{array}{c}\text { More than two } \\
\text { different movements }\end{array}$ & 15 \\
\hline & & & $\begin{array}{l}\text { Two different } \\
\text { movements }\end{array}$ & 10 \\
\hline & & & One movement & 5 \\
\hline & \multirow[t]{3}{*}{4} & \multirow[t]{3}{*}{ Visual pursuit } & On four (all) occasions & 16 \\
\hline & & & On three occasions & 12 \\
\hline & & & On two occasions & 8 \\
\hline & \multirow[t]{3}{*}{3} & \multirow[t]{3}{*}{ Visual fixation } & On four (all) occasions & 12 \\
\hline & & & On three occasions & 9 \\
\hline & & & On two occasions & 6 \\
\hline & \multirow[t]{2}{*}{2} & \multirow[t]{2}{*}{ Pain localization* } & On both hands & 4 \\
\hline & & & On one hand & 2 \\
\hline \multirow[t]{2}{*}{ UWS } & \multirow[t]{2}{*}{1} & \multirow[t]{2}{*}{ Arousal } & Spontaneously & 4 \\
\hline & & & To auditory stimulation & 3 \\
\hline
\end{tabular}




\begin{tabular}{|c|c|c|c|c|}
\cline { 3 - 4 } & & & To tactile stimulation & To pain \\
\cline { 3 - 4 } & 0 & & None \\
\hline Coma & 0 & 0 \\
\hline
\end{tabular}

Table 1. Scoring, associated diagnosis, and additional index points. Additional index points obtained for each conditional item should be added to calculate the additional index score, ranging from 0 to 100 . Zero additional index point should be scored for non-administered conditional items (e.g., pain localization when command-following is present) or unsuccessful items (i.e., when the criteria to score the item are not met). ${ }^{*}$ denotes conditional items. 
Date :

Time

Simplified Evaluation of CONsciousness Disorders (SECONDs)

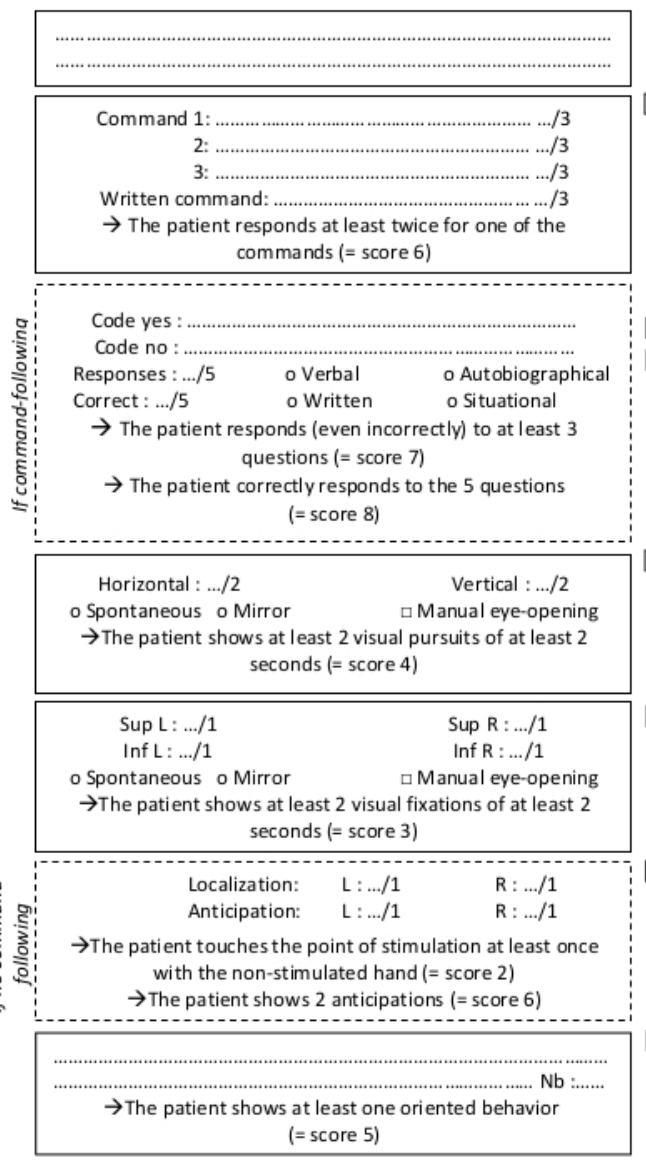

$0-25 \% / 25-50 \% / 50-75 \% / 75-100 \%$ Spontaneously / Auditory / Tactile / Pain stimulations

$\rightarrow$ The patient shows at least one eye-opening during the whole assessment (= score 1 )
A. Observation

B. Command-following (score 6)

$3 \times 3$ spoken commands

$10^{\prime \prime}$ interval between commands

$(1 \times 3$ written command if $0 / 3$ )

Stop if 2 commands $3 / 3$

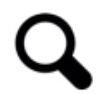

\section{Communication}

Intentional (score 7)

Functional (score 8)

Autobiographical questions

Name (no), birth date (yes), name (yes), birth date (no), children (yes/no)

If incorrect answer(s): Situational questions Place (yes), wearing a hat (no), place (no), touching hand (yes), to uching face (no)

$\square$ D. Visual pursuit (score 4)

Person/mirror, $30 \mathrm{~cm}$ from face

Each movement on horizontal

or vertical axes $=4 "(\rightarrow \leftarrow \downarrow \uparrow)$

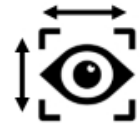

$\square$ E. Visual fixation (score 3 ) Person/mirror, $30 \mathrm{~cm}$ from face

Present stimulus in each

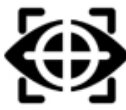

quadrant

F. Pain localization (score 2)

Inform patient

5 " pressure on nail bed

1 trial on each hand

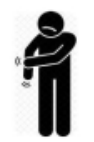

$\square$ G. Oriented behaviors (score 5)

E.g., scratching, grabbing sheets, holding bed, laughing or crying contextually,...

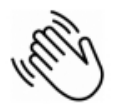

H. Arousal

$\square$ Eye opening (score 1 )

$\square$ No arousal (score 0)

Report the percentage of eyeopening time and administered stimulations

Diagnosis : Coma (0) / UWS (1) / MCS- (2-5) / MCS+ (6-7) / EMCS (8)

Additional index score : ... /100

Figure 1. Administration protocol of the SECONDs. The scale features eight items (six mandatory - A, B, D, E, G, H and two conditional - C, F) presented in the order of administration. All required fields should be completed and the highest score used to determine the patient's behavioral diagnosis. Please click here to view a larger version of this figure. 


\section{Representative Results}

Figure 1 presents the administration protocol and scoring sheet of the SECONDs. In a previous French validation study performed on 57 DoC patients ${ }^{22}$, the inclusion criteria included the presence of severe acquired brain injury, a prolonged DoC (i.e., at least 28 days since injury), minimum age of 18 years, no history of neurological or psychiatric deficit, oral fluency in French, and a stable medical condition. Three SECONDs and one CRS-R assessments were performed on two consecutive days, including randomization and blinding procedures. The administration duration of the SECONDs $($ median $=7 \mathrm{~min}$; interquartile range $=5-9 \mathrm{~min}$ ) was significantly shorter compared to the CRS-R (median $=17 \mathrm{~min}$; interquartile range $=12-22 \mathrm{~min} ; \mathrm{W}=8791, \mathrm{p}<$ 0.001). The concurrent validity was excellent for the CRS-
$\mathrm{R}$ and the SECONDs' best diagnosis $(\mathrm{KW}=0.85)$. The intra-rater reliability $\left(\mathrm{KW}_{\mathrm{W}}=0.85\right)$ and inter-rater reliability $(\mathrm{KW}=0.85)$ were also excellent. The CRS-R total score correlated with the score of the best SECONDs $\left(r_{S}=0.92\right.$; $S=2343.8, p<0.001)$. The diagnostic disagreement between the best SECONDs and the CRS-R was 11/57 (19\%) (Table 2). There was a significant negative correlation between the SECONDs administration duration and the patient level of consciousness (Spearman's rho $=-0.49, p=2.26 \times 10^{-12}$, $S=1.40 \mathrm{E} 6)$. Shorter assessments corresponded to patients with better diagnoses (median administration duration $=8 \mathrm{~min}$ for UWS patients, $7 \mathrm{~min}$ for MCS patients, $5 \mathrm{~min}$ for EMCS patients). These data tend to rule out the possibility that a longer assessment time may have increased the chance to observe conscious behaviors.

\begin{tabular}{|c|c|c|c|}
\hline & CRS-R & Same-day SECONDs & Best SECONDs \\
\hline \multirow[t]{3}{*}{ Administration duration } & Median = $17 \mathrm{~min}$ & \multirow{2}{*}{\multicolumn{2}{|c|}{ Median $=7 \mathrm{~min}, \mathrm{IQR}=5-9 \mathrm{~min}$}} \\
\hline & $I Q R=12-22 \min$ & & \\
\hline & \multicolumn{3}{|c|}{$W=8791 ; p<0.001$} \\
\hline \multicolumn{4}{|l|}{ Diagnosis } \\
\hline UWS patients & 12 & 14 & 13 \\
\hline MCS patients & 28 & 27 & 25 \\
\hline EMCS patients & 17 & 16 & 19 \\
\hline $\begin{array}{l}\text { Concurrent validity } \\
\text { with the CRS-R }\end{array}$ & & $\mathrm{KW}_{\mathrm{W}}=0.79 ; 48 / 57 ; 84.21 \%$ & $\mathrm{KW}_{\mathrm{W}}=0.84 ; 50 / 57 ; 87.72 \%$ \\
\hline Intra-rater reliability & & \multicolumn{2}{|c|}{$\mathrm{KW}_{\mathrm{W}}=0.91 ; 53 / 57 ; 92.98 \%$} \\
\hline Inter-rater reliability & & \multicolumn{2}{|c|}{$\mathrm{K}_{W}=0.82 ; 49 / 57 ; 85.96 \%$} \\
\hline
\end{tabular}


Correlation between

SECONDs and

CRS-R total scores

\begin{tabular}{|c|c|c|} 
& $r_{S}=0.91$ & $r_{S}=0.92$ \\
\hline & $S=3110.2 ; p<0.001$ & $S=2343.8 ; p<0.001$ \\
\hline
\end{tabular}

Table 2. Representative results from the SECONDs validation study ${ }^{22}$. Diagnostic assessments of patients with disorders of consciousness were compared, using either the Coma Recovery Scale - Revised (CRS-R - left column), the Simplified Evaluation of CONsciousness Disorders (SECONDs) performed on the same day as the CRS-R (middle column) or the SECONDs with the best diagnosis (right column). Administration durations were compared using a Mann Whitney $\mathrm{U}$ test (IQR = interquartile range; $\mathrm{W}=$ Mann-Whitney test statistic). Concurrent validity, intra-rater reliability and interrater reliability were calculated using Fleiss' weighted coefficients $\left(K_{W}\right)$. Spearman's rank correlation coefficients $\left(r_{s}\right)$ were computed to compare total scores of the SECONDs and the CRS-R ( $S=$ Spearman's test statistic).

To highlight the importance of a meticulous administration procedure and the need to follow the scoring guidelines thoroughly, three fictional illustrative cases are presented featuring frequent pitfalls that may be encountered while administering this scale (Figure 2). These examples are based on realistic clinical situations and aim to demonstrate how a single small deviation in the administration procedure can result in a misdiagnosis and, consequently, in inappropriate patient management. As a general rule, examiners should always attempt to elicit the best possible response for a given item (e.g., by promoting arousal, encouraging the patient, and optimizing the testing environment) but should score responses that are clear, unequivocal, and reproducible only. Any ambiguous or questionable responses should not be scored but can be mentioned as a comment and re-evaluated in future assessments.

Case 1 illustrates the importance of command selection. An examiner is called in to evaluate a 34 -year-old female patient diagnosed in UWS for several years who has begun presenting blinking, agitation, tongue protrusion, and upward eye movements since the morning. The nurse asks whether the patient could suffer from a LIS and is worried that the patient may be trying to communicate and that the patient has been conscious the whole time. The patient has been given domperidone during the night because of nausea and vomiting. Upon arrival to the room, the patient can be observed to exhibit repeated episodes of sustained upward gaze lasting around 10 seconds each. When administering the SECONDs, no response is obtained to the commands 'Move your hand', 'Open your mouth' or 'Look down'. The patient exhibits no visual pursuit or fixation, no localization to pain, and no oriented behaviors. Selection of the 'Look up' command could have fortuitously resulted in a score of 6 and therefore an erroneous diagnosis of MCS+, as spontaneous repeated movements must not be used to test commandfollowing. A correct administration of the SECONDs following the guidelines results in a score of 1 and a diagnosis of UWS. This patient is suffering from an oculogyric crisis triggered by the administration of domperidone, with typical episodes of involuntary upward bilateral elevation of the eyes.

Case 2 illustrates the importance of question selection when testing communication. A 67-year-old female patient is admitted to the emergency room hospital for acute 
ethanol consumption with respiratory failure. Now medically stable, the patient's level of consciousness must be assessed. The patient correctly responds to commands ('Squeeze my hand', 'Look up') with some delay. When communication is tested using autobiographical questions, the patient provides $5 / 5$ verbal responses but only $2 / 5$ are correct. When testing communication using the situational questions, the patient answers with $5 / 5$ correct answers. This patient suffers from Korsakoff syndrome associated with retrograde amnesia due to chronic alcohol consumption and thiamine deficiency. Testing communication using only autobiographical questions would result in a score of 7 and a diagnosis of MCS+, due to memory deficits and not altered consciousness. Correct administration of the SECONDs testing communication with both question sets would result in a correct score of 8 and a diagnosis of EMCS.

Case 3 illustrates the importance of manual eye-opening. A 50-year-old male patient is admitted to the intensive care unit for a respiratory failure due to envenomation from a cobra snake bite. He is now medically stable and his level of consciousness must be assessed, as the medical staff says the patient does not open his eyes even after withdrawal of all sedative drugs. Upon administration of the SECONDs, an absence of eye-opening despite auditory, tactile, and noxious stimulation is observed. No movement of the limbs can be observed either spontaneously or when testing command-following. However, when manually opening the eyelids, the patient shows command-following and functional communication using eye movements ('Look up for yes' and 'Look down for no'). This patient has bilateral neurotoxic ptosis and limb paralysis due to the neurotoxins contained within the venom but is fully conscious. Administration of the SECONDs without manual eye-opening would have resulted in a score of 0 , corresponding to a diagnosis of coma, while a correct administration reveals a score of 8 , which describes an emergence of the minimally conscious state. 

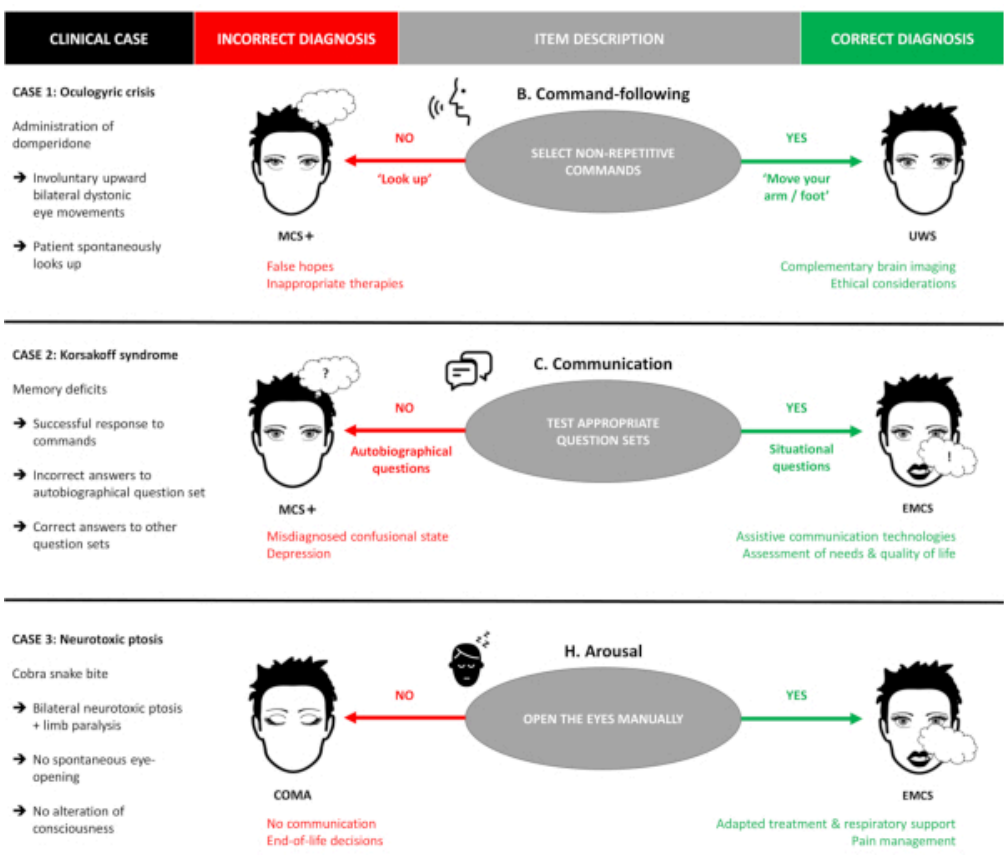

Figure 2. Illustrative clinical cases. Three clinical cases illustrating frequent administration pitfalls of the SECONDs are presented. The red column indicates the potential outcomes resulting from a suboptimal administration of the scale, while the green column describes the potential outcomes resulting from a correct administration of the SECONDs following the guidelines (e.g., in case 1, the patient is misdiagnosed as MCS+ if spontaneously repetitive commands are tested, and correctly diagnosed as UWS if appropriate non-repetitive commands are used). MCS+: minimally conscious state plus; UWS: unresponsive wakefulness syndrome; EMCS: emergence of the minimally conscious state. Please click here to view a larger version of this figure.

\section{Discussion}

The SECONDs was elaborated as a new behavioral tool inspired by the CRS-R ${ }^{16}$ and a study by Wannez et al. ${ }^{23}$ to diagnose consciousness disorders and to meet the constraints of clinical settings. The resulting illustrated administration guide provides visual targeted guidance and hands-on standardized procedures for properly administering this new behavioral scale.

\section{Advantages of the SECONDs}

The SECONDs is fast to administer and may thus allow repeated assessments even in clinical settings with important constraints. Its short duration further decreases patient fatigue and optimizes patient collaboration, potentially reducing the misdiagnosis rate in this challenging population $^{21}$. The inclusion of conditional items (i.e., communication and localization to pain) further ensures both time gain and patient compliance. Unlike the CRS-R, the SECONDs additionally provides a total score directly reflecting one diagnosis $(0=$ coma, 1 = UWS, 2-5 = MCS-, 6-7 = MCS+, $8=$ EMCS), which facilitates its interpretation. 
An additional index can be calculated to more accurately track the patient's behavioral evolution over time. Finally, this new scale offers an easy-to-use assessment that requires less material and a shorter training (provided that the administration guidelines are properly followed) compared to the CRS-R, for which the examiner's level of experience might influence the final scores ${ }^{24}$.

\section{Critical administration pitfalls of the SECONDs}

Even if the SECONDs administration is completed quickly, sufficient time should be allowed for the patient to produce the required responses. To obtain comparably high rates of diagnostic accuracy using the SECONDs, it is important that examiners dutifully follow the instructions of the administration guidelines, especially examiners without extensive experience with the CRS-R. Indeed, the administration of several SECONDs items may involve diverse pitfalls, some of which were identified in the validation study ${ }^{22}$.

\section{Command-following}

A command-following assessment is a first step toward the identification of communication abilities and the recovery of social interactions. The examiner should therefore endeavor to thoroughly assess the patient's ability to respond to commands. A particular attention should be paid to the choice of commands. The commands must be feasible for the patient and account for any (neurological, motor, linguistic, etc.) limitations. This is particularly important in cases of suspected LIS, in which at least one of the commands should be related to eye movements (e.g., 'Look up'). Indeed, the differential diagnosis between EMCS and LIS using the SECONDs is possible only if at least one oculomotor command is tested. The diagnosis can be further confirmed with the communication assessment using an adapted communication code based on eye movements, which should reveal intact cognitive functions and therefore preserved functional communication. The examiner should always keep this diagnosis in mind when assessing patients without motor response, in particular those with a lesion in the brainstem. As previously stated ${ }^{25}$, a good knowledge of the patient profile is therefore crucial. The commands should also avoid movements that are spontaneously performed at a regular rhythm, as illustrated in case 1. Importantly, such movements must be identified in the observation assessment. Finally, the commands should not require too much strength, as the patient must be able to repeat the movement.

\section{Communication}

The implementation of a "yes/no" code may be particularly difficult with some patients. It might therefore be necessary to double-check that the patient has a good understanding and ability to use the code by repeatedly asking the patient to show a "yes" and a "no". The SECONDs includes five autobiographical questions for assessing communication, as best results have previously been found using such questions $^{26}$. If the patient does not respond, the use of situational questions is proposed to consider potential severe memory deficits, as illustrated in case 2 .

\section{Visual pursuit}

This assessment should be administered very cautiously in the SECONDs. Visual pursuit is one of the most frequently observed signs of consciousness among MCS patients ${ }^{23}$ and is a common source of disagreement between the SECONDS and the CRS-R. In the SECONDs, the administration of the visual pursuit includes a criterion of time (i.e., visual pursuit duration), instead of angle amplitude, for patients 
with oculomotor impairments, which should be taken into account during the assessment. Note that this time criterion also avoids practical difficulties regarding the estimation of angular width without dedicated tools ${ }^{27}$. Furthermore, the absence of visual pursuit in a single assessment does not necessarily mean that the patient is unable to perform this item. Indeed, this assessment requires an effective eyeopening in addition to a good attentional capacity. Arousal should be promoted before starting the assessment, and manual eye-opening should be used when spontaneous eyeopening is not sustained or is insufficient to properly test visual pursuit, as demonstrated in case 3.

\section{Applicability of the SECONDs}

This tool was designed to assess consciousness among a broad population of subjects with various clinical conditions and its wide applicability allows an examiner to monitor the effect of an intervention, treatment, or complication on the level of consciousness of the patient. Special attention should be paid to any change in the treatment regimen of a patient between two consecutive SECONDs, as psychoactive medication could have an influence on the observed score. To maximize reproducibility of the results, assessing patients with stable vital parameters, who are off sedative drugs, and who are on a stable dosage of necessary treatments with potential psychoactive activity (e.g., antiepileptic drugs) are recommended. Administering the SECONDs right after a straining examination or therapy (e.g., MRI, physiotherapy) should also be avoided. The interpretation of the results should take these confounding factors into consideration when they cannot be eluded. Repeated assessments are particularly encouraged when optimal administration conditions are not met and complementary diagnostic techniques should be used in combination with the SECONDs. In particular, end-of-life decisions should always be based on the most thorough and accurate tools available, including repeated standardized behavioral scales and validated paraclinical measures of brain function. In that sense, the CRS-R should be preferred to the SECONDs in all cases where a more granular and comprehensive clinical assessment of consciousness is needed. In research settings, the SECONDs should be considered in protocols in which the administration of the full CRS-R is not possible due to practical or logistical reasons (e.g., a study investigating vigilance fluctuations that requires repeated assessments within the same day). Additionally, the SECONDs was not designed to accurately monitor the presence of reflexive behaviors. In patients with a diagnosis of UWS or coma, brainstem reflexes and other primitive neurological signs should also be tested, as they provide valuable pathophysiological and prognostic information. Basic information can be obtained with the widely used Glasgow Coma Scale (GCS) ${ }^{28}$ and additional elements can be assessed using more detailed clinical scales, such as the CRS-R ${ }^{16}$, the Coma/Near Coma Scale ${ }^{29}$, the Full Outline of UnResponsiveness (FOUR) ${ }^{30}$, or the GlasgowLiège Scale ${ }^{31}$.

\section{Future applications}

As the original validation study was conducted in Frenchspeaking patients, several undergoing studies will propose a translation of this new scale into English and other languages. Future work should also focus on an external validation in a larger sample and include repeated SECONDs versus CRS-R evaluations as was previously recommended ${ }^{21,32}$. The SECONDs and its index score should additionally be validated in acute settings (i.e., intensive care units) and compared to other scales such as the $\mathrm{GCS}^{28}$ and the 
FOUR $^{30}$. In this view, a prospective longitudinal study should also assess its predictive value regarding the degree of functional recovery, using appropriate rehabilitation scales, as previously demonstrated for the CRS-R ${ }^{33}$. Given that the SECONDs is an easy-to-use tool requiring minimal training, family members could easily be implicated in the diagnosis of their relatives with DoC. As the scale was validated on an adult population, additional studies are needed to determine its applicability among children. Neurophysiology and neuroimaging techniques play a major role in the modern assessment of DoC. As the SECONDs can be easily administered before or after paraclinical tests (e.g. EEG, MRI or PET), investigating the relationships between this new scale and specific biomarkers may also provide interesting diagnostic and prognostic perspectives. These future essential validation steps will involve multiple healthcare staff members from several teams across the world with contrasting experience in the multimodal assessment of patients with severe brain injuries.

\section{Conclusion}

The SECONDs is a quick and promising tool for assessing the level of consciousness in severely brain-injured patients. This new scale can easily be implemented in clinical and research settings to reduce misdiagnoses and, consequently, optimize end-of-life and therapeutic decisions in this challenging population. The use of this administration guide will facilitate its implementation among untrained professionals and improve its reproducibility across examiners.

\section{Disclosures}

The authors have nothing to disclose.

\section{Acknowledgments}

We thank the teams from the Intensive Care Department and the Neurology Department of the University Hospital of Liège, CHN W. Lennox Center (David Dikenstein and colleagues), ISoSL Valdor Hospital (Dr Haroun Jedidi and colleagues), and ACSOL Bouge Center (Kathy Costabeber and colleagues), as well as the patients and their families.

The study was supported by the University and University Hospital of Liège, the French Speaking Community Concerted Research Action (ARC 12-17/01), the Belgian National Funds for Scientific Research (F.R.S-FNRS), the Generet funds and King Baudouin Foundation, the Marie Sklodowska-Curie Actions (H2020-MSCA-IF-2016ADOC-752686), the European Union's Horizon 2020 Framework Program for Research and Innovation under the Specific Grant Agreement No. 945539 (Human Brain Project SGA3), the James McDonnell Foundation, the Mind Science Foundation, IAP research network P7/06 of the Belgian Government (Belgian Science Policy), the European Commission, the Public Utility Foundation 'Université Européenne du Travail', the "Fondazione Europea di Ricerca Biomedica", the BIAL Foundation, the AstraZeneca Foundation, and the Belgian National Plan Cancer (139). C.A., L.S. and N.L. are research fellows, A.T. is a postdoctoral fellow, O.G. is research associate, and S.L. is research director at the F.R.S-FNRS.

\section{References}

1. Laureys, S., Owen, A.M., Schiff, N.D. Brain function in coma, vegetative state, and related disorders. Lancet Neurology. 3 (9), 537-546 (2004).

2. Laureys, S. et al. Unresponsive wakefulness syndrome: A new name for the vegetative state or apallic syndrome. BMC Medicine. 8, 2-5 (2010). 
3. The Multy-Society Task Force on PVS Medical aspects of the persistent vegetative state (2). The New England Journal of Medicine. 330 (22), 1499-1508 (1994).

4. Giacino, J.T., et al. The minimally conscious state. Neurology. 58 (3), 349-353 (2002).

5. Bruno, M.-A.A., Vanhaudenhuyse, A., Thibaut, A., Moonen, G., Laureys, S. From unresponsive wakefulness to minimally conscious PLUS and functional locked-in syndromes: recent advances in our understanding of disorders of consciousness. Journal of Neurology. 258 (7), 1373-1384 (2011).

6. Bruno, M.A., Laureys, S., Demertzi, A. Coma and disorders of consciousness. Handbook of Clinical Neurology. 118, 205-213 (2013).

7. Thibaut, A., Bruno, M.-A., Ledoux, D., Demertzi, A., Laureys, S. tDCS in patients with disorders of consciousness Sham-controlled randomized doubleblind study. Neurology. 82 (13), 1112-1118 (2014).

8. Chatelle, C. et al. Is the Nociception Coma Scale-revised a Useful Clinical Tool for Managing Pain in Patients With Disorders of Consciousness? The Clinical Journal of Pain. 32 (4), 321-326 (2016).

9. Estraneo, A. et al. Multi-center prospective study on predictors of short-term outcome in disorders of consciousness. Neurology. (2020).

10. Demertzi, A. et al. Attitudes towards end-of-life issues in disorders of consciousness: A European survey. Journal of Neurology. 258 (6), 1058-1065 (2011).

11. Andrews, K., Murphy, L., Munday, R., Littlewood, C. Misdiagnosis of the vegetative state: retrospective study in a rehabilitation unit. The BMJ (Clinical research ed.). 313 (7048), 13-6 (1996).
12. Childs, N.L., Mercer, W.N., Childs, H.W. Accuracy of diagnosis of persistent vegetative state. Neurology. 43 (8), 1465-7 (1993).

13. Gill-Thwaites, H. Lotteries, loopholes and luck: misdiagnosis in the vegetative state patient. Brain injury. 20 (13-14), 1321-8 (2006).

14. van Erp, W.S., et al. The Vegetative State: Prevalence, Misdiagnosis, and Treatment Limitations. Journal of the American Medical Directors Association. 16 (1), 85.e9-85.e14 (2015).

15. Schnakers, C. et al. Diagnostic accuracy of the vegetative and minimally conscious state: Clinical consensus versus standardized neurobehavioral assessment. 5, 1-5 (2009).

16. Giacino, J.T., Kalmar, K., Whyte, J. The JFK Coma Recovery Scale-Revised: Measurement characteristics and diagnostic utility. Archives of Physical Medicine and Rehabilitation. 85 (12), 2020-2029 (2004).

17. Seel, R.T. et al. Assessment scales for disorders of consciousness: Evidence-based recommendations for clinical practice and research. Archives of Physical Medicine and Rehabilitation. 91 (12), 1795-1813 (2010).

18. Bodien, Y.G., Carlowicz, C.A., Chatelle, C., Giacino, J.T. Sensitivity and Specificity of the Coma Recovery Scale-Revised Total Score in Detection of Conscious Awareness. Archives of Physical Medicine and Rehabilitation. 97 (3), 490-492 (2016).

19. Chatelle, C. et al. Detection and Interpretation of Impossible and Improbable Coma Recovery ScaleRevised Scores. Archives of Physical Medicine and Rehabilitation. 97 (8), 1295-1300.e4 (2016). 
20. Annen, J. et al. Diagnostic accuracy of the CRS-R index in patients with disorders of consciousness. Brain Injury. 33 (11), 1409-1412 (2019).

21. Wannez, S., Heine, L., Thonnard, M., Gosseries, O., Laureys, S. The repetition of behavioral assessments in diagnosis of disorders of consciousness. Annals of Neurology. 81 (6), 883-889 (2017).

22. Aubinet, C. et al. Simplified Evaluation of CONsciousness Disorders (SECONDs) in individuals with severe brain injury: a validation study. Annals of physical and rehabilitation medicine. 1-31 (2020).

23. Wannez, S. et al. Prevalence of coma-recovery scalerevised signs of consciousness in patients in minimally conscious state. Neuropsychological Rehabilitation. 2011, 1-10 (2017).

24. Løvstad, M., et al. Reliability and diagnostic characteristics of the JFK Coma Recovery ScaleRevised: Exploring the influence of raters level of experience. Journal of Head Trauma Rehabilitation. 25 (5), 349-356 (2010).

25. Gosseries, O., Zasler, N.D., Laureys, S. Recent advances in disorders of consciousness: Focus on the diagnosis. Brain Injury. 28 (9), 1141-1150 (2014).

26. Nakase-Richardson, R., Yablon, S.A., Sherer, M., Nick, T.G., Evans, C.C. Emergence from minimally conscious state: Insights from evaluation of posttraumatic confusion. Neurology. 73 (14), 1120-1126 (2009).

27. Wannez, S. et al. Objective assessment of visual pursuit in patients with disorders of consciousness: an exploratory study. Journal of Neurology. 264 (5), 928937 (2017).
28. Teasdale, G., Jennett, B. Assessment of coma and impaired consciousness. The Lancet. 304 (7872), 81-84 (1974).

29. Rappaport, M., Dougherty, A.M., Kelting, D.L. Evaluation of coma and vegetative states. Archives of Physical Medicine and Rehabilitation. 73 (7), 628-34 (1992).

30. Wijdicks, E.F.M., Bamlet, W.R., Maramattom, B. V., Manno, E.M., McClelland, R.L. Validation of a new coma scale: The FOUR score. Annals of Neurology. 58 (4), 585-593 (2005).

31. Born, J.D., Albert, A., Hans, P., Bonnal, J. Relative Prognostic Value of Best Motor Response and Brain Stem Reflexes in Patients with Severe Head Injury. Neurosurgery. 16 (5), 595-601 (1985).

32. Giacino, J.T. et al. Practice guideline update recommendations summary: Disorders of consciousness. Neurology. 91 (10), 450-460 (2018).

33. Portaccio, E. et al. Improvement on the Coma Recovery Scale-Revised During the First Four Weeks of Hospital Stay Predicts Outcome at Discharge in Intensive Rehabilitation After Severe Brain Injury. Archives of Physical Medicine and Rehabilitation. 99 (5), 914-919 (2018). 